WS095:

\title{
Potential of Renewable Energies and Strategies to use them
}

\author{
By
}

Hani El Nokraschy

\section{Abstract:}

A glance at the possible renewable energies in the world gives immediately the impression that their potential is probably sufficient to cover the global demand of humanity, for example: the deserts of the world receive in 6 hours more energy from the sun than the humanity consumes in a year. The reason that this huge potential was not used efficiently till now lies in the absence of technologies to convert the available natural energy to a useful form like electricity. Since about 40 years several technologies immerged that can convert efficiently natural energies to electricity, overcoming the problems of natural fluctuations is still a challenge. The presentation describes a method to convert direct sunrays, all year available in desert regions, to high quality electricity that can be supplied on demand at any time. The key issue is that the sunrays are concentrated on a focus, thus obtaining the advantage of deploying both main components of the sunrays: light and heat. Heat, when concentrated, can reach temperatures of several hundreds degrees centigrade, which is consequently stored in a mixture of molten salts. The stored heat energy - equivalent to an oil tank is then used to generate steam - equivalent to a fossil fired boiler. The steam drives in turn a conventional steam turbine, just on demand, day or night and especially in the peak hours. The costs of such technology are affordable since no fuel will be purchased, more over no negative effects will occur from emissions.

- Nokraschy Engineering, Germany 